\title{
INFLUENCIA DE LA TEXTURA DEL SUELO EN LOS BULBOS HÚMEDOS DESARROLLADOS EN SUELO ENARENADO CON EMISORES DE RIEGO LOCALIZADO DE BAJO CAUDAL
}

\author{
Zapata Sierra, A.J.(1) (P), Contreras París, J.I.(2), Martín Usero, F. (1), Baeza Cano, R. (2)
}

\author{
${ }^{1}$ Departamento de Ingeniería, Universidad de Almería, Ctra. De Sacramento s/n, 04120 \\ Almería. \\ ajzapata@ual.es \\ ${ }^{2}$ Instituto de Investigación y Formación Agraria y Pesquera de Andalucía (IFAPA), Centro La \\ Mojonera, Camino San Nicolás, nº1, 04745 La Mojonera, Almería. \\ rafaelj.baeza@juntadeandalucia.es
}

\begin{abstract}
Resumen
Se ha medido la distribución de la humedad en 20 suelos, en sistema enarenado en la comarca Campo de Dalías (Almería), regados por goteo y bajo cultivo de pimiento. Se utilizaron goteros de caudal $3 \mathrm{I} . \mathrm{h}^{-1}$ con una densidad de 2 emisores. $\mathrm{m}^{-2}$ y un tiempo de riego de 30 minutos. Se ha encontrado que existe una gran heterogeneidad en las propiedades físicas de los suelos a pesar de tratarse de suelos artificialmente aportados. El factor más importante para explicar las diferencias en la distribución de humedad fue la textura del suelo. De los ensayos se deduce que existen problemas en la distribución del riego debido a la presencia de horizontes impermeables. Por otra parte los niveles de humedad encontrados antes de los riegos sugieren que se está regando en exceso los suelos y que el área mojada alcanza a casi la totalidad de la superficie de cultivo.
\end{abstract}

\begin{abstract}
We measured the moisture distribution in 20 soils under "arenado" system in the county Campo de Dalías (Almeria), drip irrigated and a pepper crop. Flow drippers 3l.h ${ }^{-1}$ were used with a density of 2 emiters. $\mathrm{m}^{-2}$ and a run time of 30 minutes. It has been found that there is great heterogeneity in the physical properties of soils despite being artificially supplied ones. The most important factor in explaining differences in moisture distribution was the soil texture. Trials it appears that there are problems in the distribution of irrigation due to the presence of impermeable layers. Moreover moisture levels found prior to suggest that the soils are being watered excessively and the wet area reaches almost the entire culture surface.
\end{abstract}

\section{1-Introducción y objetivos}

La forma y dimensiones del volumen mojado o bulbo húmedo dependen, ante todo, de las propiedades y características del perfil físico del suelo, del volumen de agua aplicado, caudal del emisor, contenido de agua presente en el suelo al inicio de la irrigación y topografía del terreno (Lubana y Narda, 1998).

Según se recoge en la bibliografía, a medida que aumenta el caudal del emisor hay mayor predominio del desplazamiento horizontal respecto del vertical (Bresler, 1977). Para varios caudales y distintos suelos, el desplazamiento horizontal y vertical del frente de humedad crecen de forma más rápida al inicio del riego y de forma más atenuada a medida que aumenta el tiempo de aplicación. No obstante existe información contradictoria al respecto que indica que con emisores de ultrabajo caudal $(<1 \mathrm{l} / \mathrm{hora})$ hay un mayor 
desplazamiento horizontal del frente humedecido cuando se comparan con emisores de mayor caudal.

En cualquier caso, el primer factor a considerar es el perfil físico del suelo o conjunto de propiedades físicas que intervienen en el movimiento del agua en este suelo, para cada uno de los horizontes o capas, pues el grado de estratificación que presenta el perfil es de gran importancia (Lubana y Narda, 2001). De las propiedades físicas del suelo, la textura es la más influyente en el movimiento del agua en el suelo (Fuentes, 1998)y su determinación en laboratorio es sencilla.

En los cultivos hortícolas del Sureste de España se ha extendido desde hace décadas un perfil característico de suelos: el enarenado, consistente en la aplicación de una capa de arena de unos 8-10 cm de espesor sobre otra de tierra fértil de cañada, también aportada, de un espesor variable: 20-40 cm (Lao y Jiménez, 2002).

La capa superficial de arena actúa como aislante del suelo, tanto térmico, como hídrico, evitando la evaporación directa desde el suelo e influyendo en el reparto de humedad en el mismo. Existen algunos trabajos en condiciones de laboratorio(i.e. Boswel,1989; Keller y Bliesner, 2005), pero hasta la fecha, el bulbo húmedo en el perfil del suelo enarenado, utilizado mayoritariamente en los cultivos hortícolas de invernadero en la provincia de Almería, no ha sido determinado in situ.

El objetivo del presente trabajo ha sido caracterizar las dimensiones y la evolución del movimiento del agua en los bulbos húmedos generados en suelos enarenados de la comarca del Campo de Dalías con los habituales emisores de riego localizado de $3 \mathrm{I} \cdot \mathrm{h}^{-1}$ de caudal nominal. Adicionalmente el estudio permitirá caracterizar los suelos enarenados de la Comarca del Campo de Dalías.

\section{2- Material y Métodos}

Se han seleccionado 20 fincas comerciales de cultivo de pimiento (cultivo mayoritario en la zona) que cumplían con las siguientes premisas de partida:

- Suelo enarenado

- Emisores de caudal nominal: $3 \mathrm{I} \cdot \mathrm{h}^{-1}$

- Marco de riego: 2 emisores $\cdot \mathrm{m}^{-2}$ (aproximadamente)

- Tiempo de riego (dotación): 30 minutos de aplicación

- Cultivo con más de un mes de desarrollo

La selección de las fincas se realizó de forma dirigida para poder obtener información de los principales suelos de la comarca.

- Humedad inicial (previa al riego). Se midió la humedad en la línea perpendicular a los ramales de riego, coincidente con los puntos de emisión, en la línea perpendicular a los ramales, a $1 / 4$ del punto de emisión (12,5 cm respecto al gotero) y en la línea perpendicular a los ramales, a $1 \frac{1}{2}$ del punto de emisión ( $25 \mathrm{~cm}$ respecto al gotero). Se midió cada $20 \mathrm{~cm}$ en la horizontal y a tres profundidades: 10,20 y $30 \mathrm{~cm}$ (variables dependiendo de la profundidad del suelo estudiado). Se realizaron en total 60-70 medidas por par de emisores (líneas de riego pareadas). Antes de realizar las medidas se apartó la capa de arena de la zona a muestrear y se calibraron los aparatos de medición en saturación y al aire. 

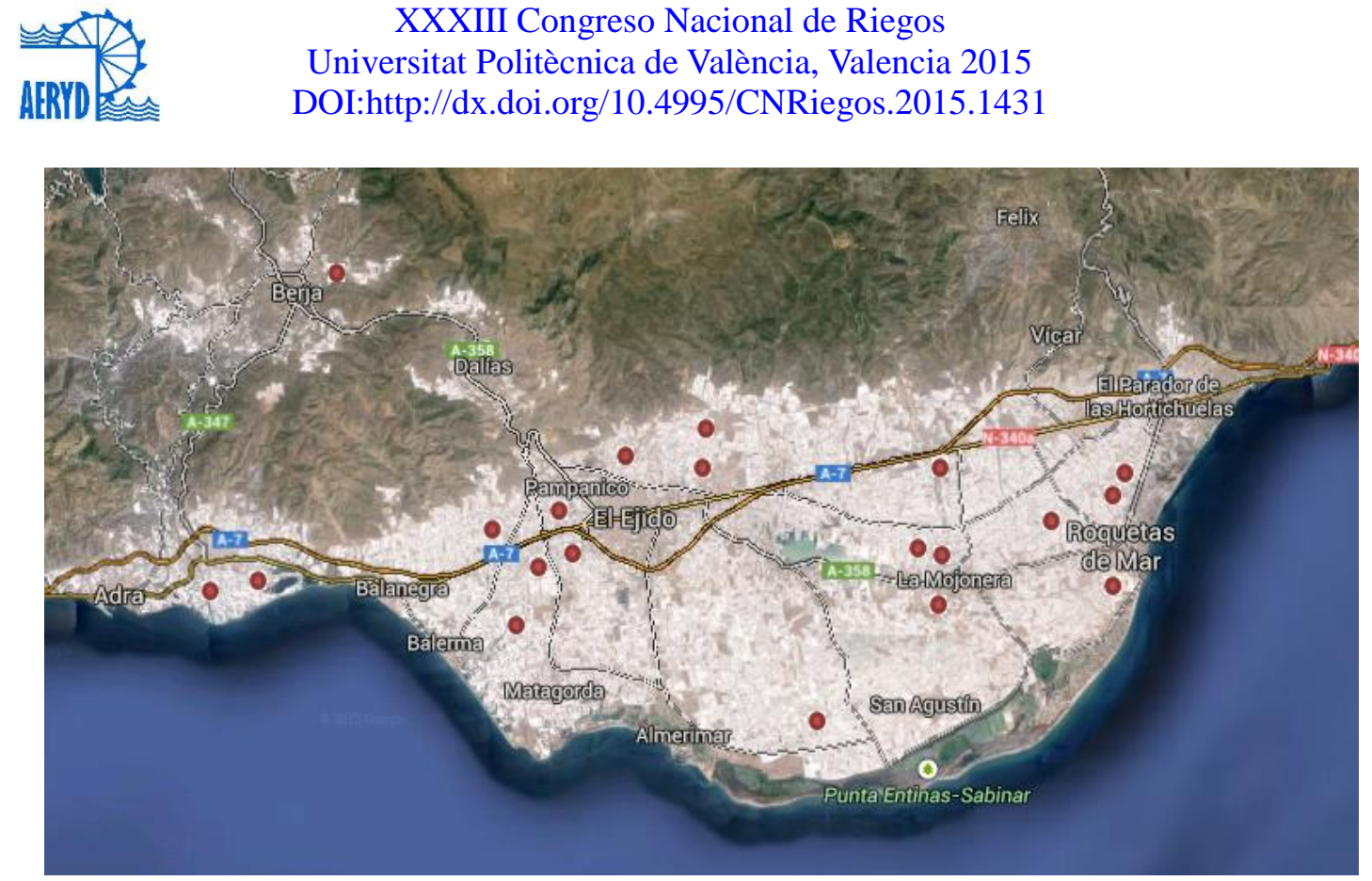

Figura 1 Distribución de los invernaderos muestreados.

- Humedad final (tras el riego). Una vez finalizado el muestreo inicial se extendió de nuevo la arena y se realizó un riego de equivalente a 1,5 I (30 minutos en emisores $\left.3 \mathrm{l} \cdot \mathrm{h}^{-1}\right)$. Al iniciar el riego se midió la presión en el ramal de medida para posteriormente calcular el caudal exacto que emiten los dos goteros de la zona de estudio. Trascurridos 5 minutos tras la finalización del riego se apartó de nuevo la arena y se iniciaron las medidas 10 minutos después (15 minutos tras el riego). Se midió en las mismas perpendiculares respecto a los ramales y a las mismas profundidades, en puntos equidistantes en la horizontal respecto a los puntos muestreados en la humedad inicial. De nuevo se realizaron un total de 60-70 medidas por par de emisores.

Los aparatos utilizados para realizar las mediciones en campo fueron: Medidor de humedad volumétrica Digital Aquaterr-M350 y el medidor analógico Spectrum Soil Moisture Tester. La medida obtenida, fracción de saturación, es convertida a humedad volumétrica.
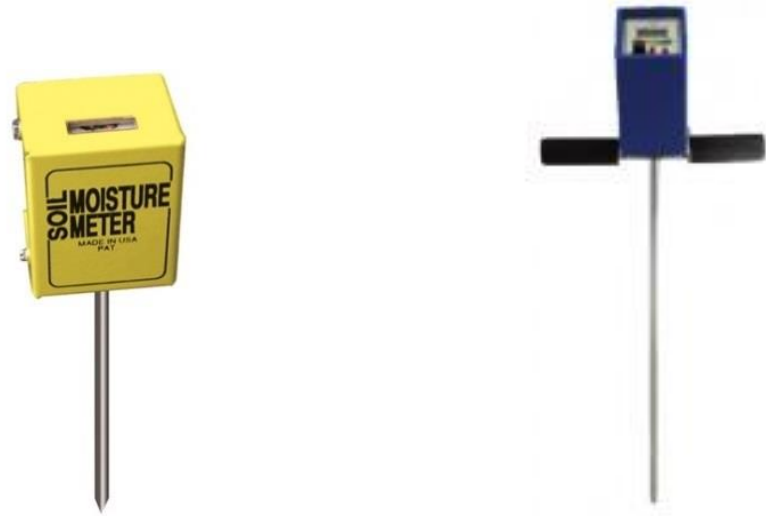

Figura 2 Aparato de medida de humedad analógico Spectrum $\AA^{\circledR}$ SoilMoistureTester.y Aparato de medida de humedad digital Aquaterr ${ }^{\circledR}$ M-350

Una vez finalizadas las mediciones en campo se extrajeron 5 cilindros de suelo para el análisis de humedad volumétrica en laboratorio. Las muestras se tomaron entre dos puntos de similar humedad medida "in situ". Para ello se eligieron 2 puntos de extracción de menor valor de humedad, dos puntos de extracción de mayor valor y un punto de valor intermedio. La humedad medida en laboratorio nos permitirá ajustar las escalas de humedad de los aparatos. 
- Textura y pedregosidad. Una vez finalizada la medición de humedad volumétrica se mezclaron las muestras de los 5 cilindros y se tomó una muestra para el análisis de textura y pedregosidad en laboratorio.

- Caudal de los emisores. Concluidas las mediciones y toma de muestras en campo se determinó el caudal exacto de los dos emisores evaluados en banco de ensayo.

\section{Análisis estadístico}

Los datos fueron analizados estadísticamente mediante un análisis de la varianza factorial (ANOVA), atendiendo a un diseño factorial con cuatro factores y estudiando la interacción entre ellos. Los datos en porcentaje fueron transformados mediante la función $1 / \sqrt{ }$ seno para obtener una distribución normal. Cuando el análisis de la varianza fue significativo, se aplicó el test de comparación de medias de mínima diferencia significativa (LSD para $P \leq 0,05 ; P \leq 0,01 ; P \leq 0,001$ ) para identificar las diferencias entre valores medios. El análisis estadístico fue realizado con el programa Statgraphics Plus v. 5.1 (Statgraphics, Warrenton, Va.).

\section{3- Resultados y conclusiones}

\subsection{Caracterización de los suelos estudiados}

Todos los suelos estudiados tienen como nexo de unión el sistema enarenado, sin embargo existen importantes diferencias físicas entre los mismos en aspectos como la textura, profundidad y capacidad de drenaje.

Tabla 1 Características de los suelos muestreados

\begin{tabular}{llcccc} 
Localización & Textura & $\begin{array}{c}\text { Arena } \\
\text { (\%) }\end{array}$ & $\begin{array}{c}\text { Limo } \\
\text { (\%) }\end{array}$ & $\begin{array}{c}\text { Arcilla } \\
\text { (\%) }\end{array}$ & $\begin{array}{c}\text { Grava } \\
\text { (\%) }\end{array}$ \\
\hline Invernadero 1 & Franco-arenoso & 73,8 & 13,8 & 12,5 & 47,1 \\
Invernadero 2 & Franco-arcillo-arenoso & 62,5 & 13,8 & 23,8 & 24,2 \\
Invernadero 3 & Franco-arcillo-arenoso & 68,8 & 11,3 & 20,0 & 19,0 \\
Invernadero 4 & Franco-arcillo-arenoso & 65,0 & 12,5 & 22,5 & 48,7 \\
Invernadero 5 & Franco-arenoso & 66,3 & 20,0 & 13,8 & 9,3 \\
Invernadero 6 & Arenoso-franco & 83,8 & 7,5 & 8,8 & 42,6 \\
Invernadero 7 & Franco-arcillo-arenoso & 51,3 & 17,5 & 31,3 & 21,4 \\
Invernadero 8 & Franco-arcilloso & 43,8 & 25,0 & 31,3 & 4,3 \\
Invernadero 9 & Franco-arcillo-arenoso & 68,8 & 6,3 & 25,0 & 3,4 \\
Invernadero 10 & Arenoso-franco & 83,8 & 3,8 & 12,5 & 18,1 \\
Invernadero 11 & Franco-arcilloso & 33,8 & 32,5 & 33,8 & 14,1 \\
Invernadero 12 & Franco-arcillo-arenoso & 52,5 & 18,8 & 28,8 & 30,4 \\
Invernadero 13 & Franco-arenoso & 65,0 & 17,5 & 17,5 & 16,3 \\
Invernadero 14 & Arcilloso & 30,0 & 23,8 & 46,3 & 38,4 \\
Invernadero 15 & Franco-arcilloso & 40,0 & 31,3 & 28,8 & 28,2 \\
Invernadero 16 & Arenoso-franco & 80,0 & 11,3 & 8,8 & 38,3 \\
Invernadero 17 & Arcilloso & 27,5 & 20,0 & 52,5 & 10,9 \\
Invernadero 18 & Arcilloso & 20,0 & 30,0 & 50,0 & 2,1 \\
Invernadero 19 & Franco-arcillo-arenoso & 76,3 & 7,5 & 16,3 & 11,3 \\
Invernadero 20 & Franco-arcillo-arenoso & 57,5 & 18,8 & 23,8 & 12,9 \\
\hline
\end{tabular}


Tanto la textura como el porcentaje de grava se refieren al horizonte de tierra aportada o no que subyace bajo la capa de arena y la de materia orgánica que conforman el suelo enarenado.

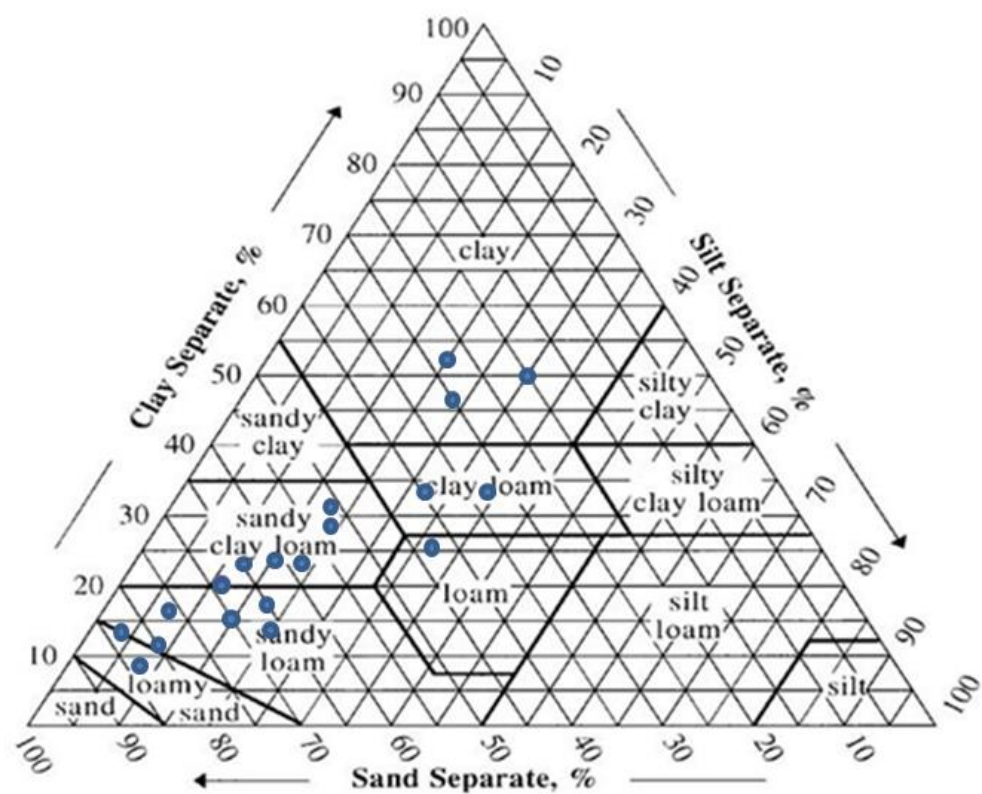

Figura 3 Diagrama triangular de texturas de los suelos analizados

\subsection{Bulbos húmedos generados}

Un $30 \%$ de los suelos estudiados mostraban niveles de humedad muy elevados antes de iniciar el riego y el incremento de humedad en los mismos tras aplicar el mismo es mínimo, lo que indica que se está regando en exceso. En este sentido se puede ver la Figura 4. 

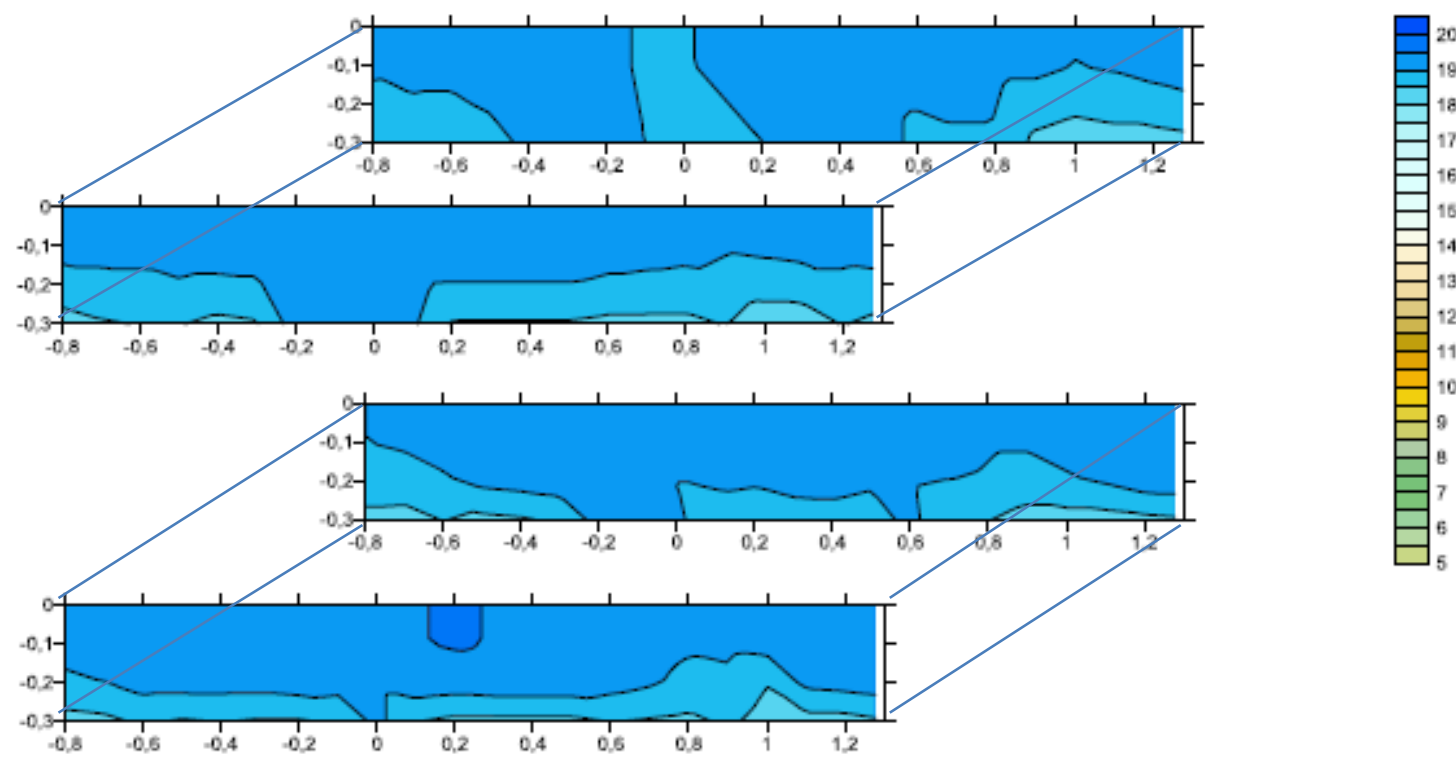

Figura 4 Perfil de distribución de la humedad en el suelo del Invernadero1, antes (arriba) y después de la operación de riego (abajo). Se muestra al fondo el perfil en la línea del gotero y delante el perfil de la línea media entre goteros

Antes de aplicar el pulso de riego, la mayor parte de los suelos evaluados presentan los niveles de humedad más bajos en la zona más cercana al emisor, coincidente con la zona de mayor concentración radicular. Se presenta la Figura 5 para mostrar este caso.
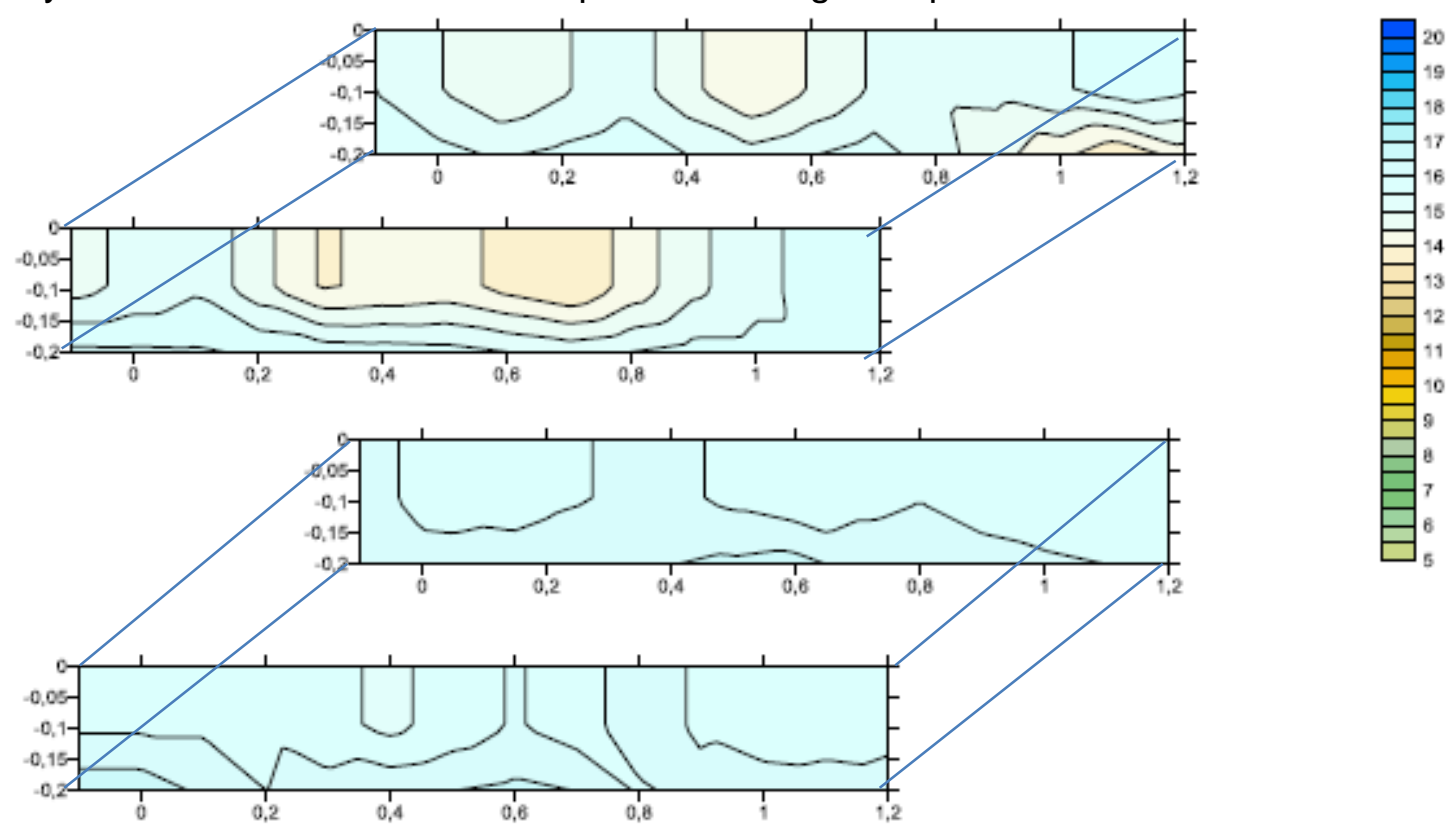

Figura 5 Perfil de distribución de la humedad en el suelo del Invernadero 11, antes (arriba) y después de la operación de riego (abajo). Se muestra al fondo el perfil en la línea del gotero y delante el perfil de la línea media entre goteros

Alguno de los suelos evaluados muestra niveles de humedad más elevados en la capa inferior del suelo, lo que denota la presencia de capas inferiores impermeables, muy frecuentes en la comarca. Para ilustrar este caso se muestra la Figura 6. 

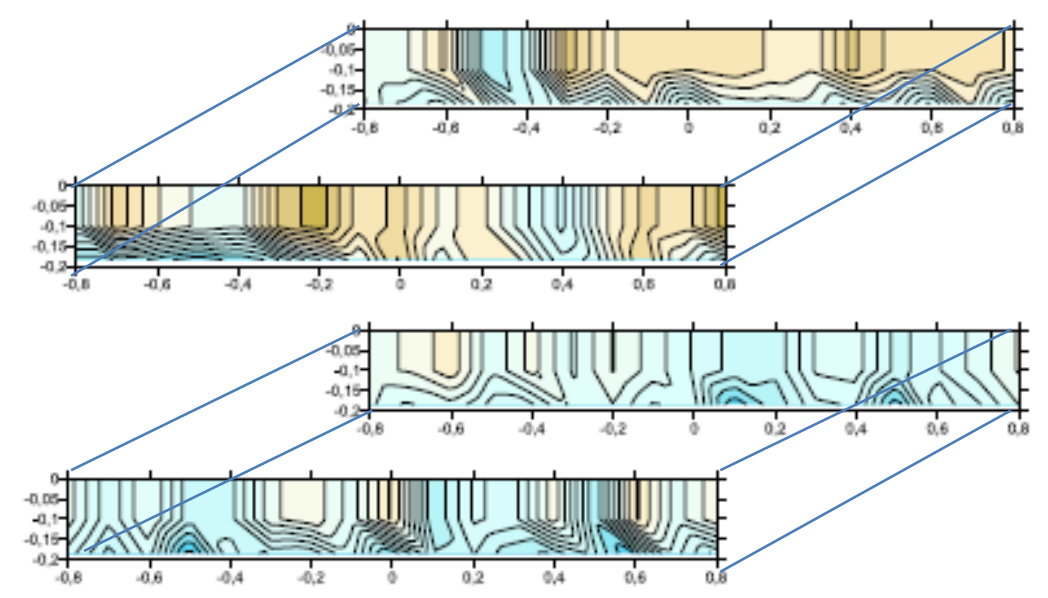

Figura 6 Perfil de distribución de la humedad en el suelo del Invernadero 8, antes (arriba) y después de la operación de riego (abajo). Se muestra al fondo el perfil en la línea del gotero y delante el perfil de la línea media entre goteros.

En la mayor parte de los suelos la distribución de humedad en la horizontal es uniforme, tanto en la línea de emisión como en la perpendicular a la misma. En estos casos el marco de riego y caudal de riego consigue el solape entre emisores de la misma línea y entre líneas adyacentes. Se puede concluir que se riega en toda la superficie del suelo. Únicamente en los suelos con porcentajes de arcilla inferiores al 15\% se aprecian zonas del terreno no humectadas. En este sentido se ofrece la Figura 7.

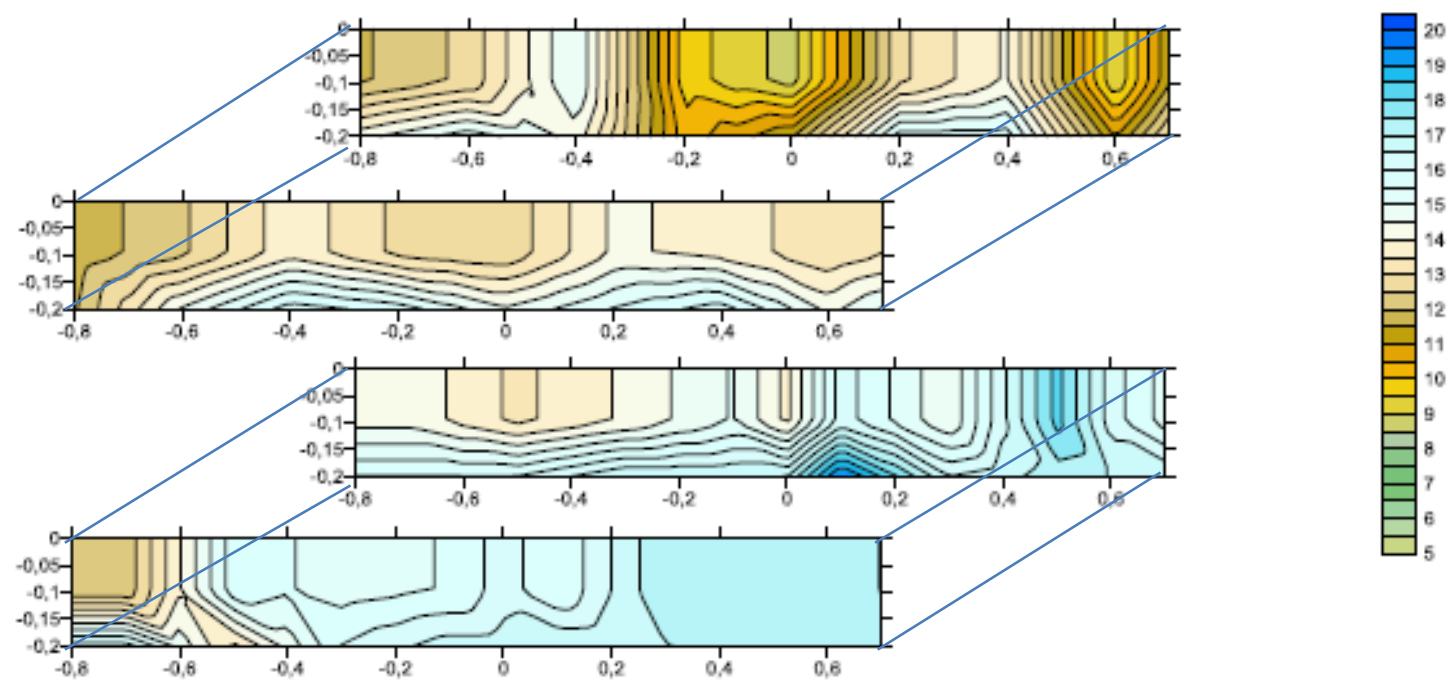

Figura 7 Perfil de distribución de la humedad en el suelo del Invernadero 14, antes (arriba) y después de la operación de riego (abajo). Se muestra al fondo el perfil en la línea del gotero y delante el perfil de la línea media entre goteros

\subsection{Efecto de los factores estudiados en el nivel de humedad del suelo}

El análisis factorial mostró que la textura es el factor de mayor influencia en el nivel de humedad del suelo, tanto antes del riego como después, afectando también el incremento de humedad tras el riego (Tabla 2). 
Antes del riego, los suelos arcillosos (arcilloso y franco-arcilloso) fueron los que mayor nivel de humedad volumétrica presentaban, con valores medios de $15,5 \%$ en el perfil del suelo. Los que menor porcentaje de humedad volumétrica presentaron fueron los suelos arenoso-francos con un valor de 10,1\%. Después del riego, aunque los valores de humedad fueron mayores, el efecto de la textura siguió siendo el mismo (Tabla 3).

El nivel de humedad volumétrica también se vio afectado por la profundidad, tanto antes como después del riego (Tabla 2). Antes del riego, la mayor humedad se detectaba a mayor profundidad $(-30 \mathrm{~cm})$ y la menor a $-10 \mathrm{~cm}$ (Tabla 4) con valores medios de $13,5 \%$. Después del riego estas diferencias se atenúan (Tabla 4).

El análisis estadístico reveló interacciones en el incremento de humedad entre la textura y la profundidad del suelo, y entre la textura y la línea del suelo medida (Tabla 2). La interacción entre la textura y la profundidad del suelo se muestra en la Figura 8. En los suelos arcillosos no existieron diferencias en el incremento de humedad del suelo en profundidad, presentando tanto a 10,20 y $30 \mathrm{~cm}$ unos valores muy similares sin diferencias estadísticamente significativas (Figura 8), igual sucede en los suelos francos (francoarcillosos, franco-arcillo-arenosos y franco-arenosos). En suelos de textura arenosa-franca se muestran diferencias significativas entre los niveles de humedad registrados a 10, 20 y 30 $\mathrm{cm}$ de profundidad, presentando a $10 \mathrm{~cm}$ el mayor incremento de humedad (Figura 8).

Tabla 2 Nivel de significación de los factores estudiados e interacciones entre factores. Incremento de humedad registrado tras el riego

\begin{tabular}{|c|c|c|c|}
\hline & \multicolumn{3}{|c|}{ Humedad del suelo } \\
\hline & Antes del riego & Después del riego & Incremento $^{1}$ \\
\hline \multicolumn{4}{|l|}{ Factor } \\
\hline A: Textura & *** & *** & *** \\
\hline B: Profundidad & *** & * & ns \\
\hline C: Línea & ns & ns & ns \\
\hline D: Distancia a gotero & ns & ns & ns \\
\hline \multicolumn{4}{|l|}{ Interacción } \\
\hline$A B$ & ns & ns & *** \\
\hline$A C$ & ns & ns & * \\
\hline AD & ns & ns & ns \\
\hline$B C$ & ns & ns & ns \\
\hline BD & ns & ns & ns \\
\hline CD & ns & ns & ns \\
\hline ABC & ns & ns & * \\
\hline ABD & ns & ns & ns \\
\hline ACD & ns & ns & ns \\
\hline BCD & ns & ns & ns \\
\hline
\end{tabular}

ns, no significativo.

*Significativo para $P<0.05$.

${ }^{* *}$ Significativo para $P<0.01 . \quad{ }^{* *}$ Significativo para $P<0.001$.

Tabla 3 Efecto de la textura del suelo en el porcentaje de humedad volumétrica. ARE-F: Arenoso-franco; F-ARC-ARE: Franco-arcillo-arenoso; F-ARE: Franco-arenoso; F-ARC: Franco-arcilloso; ARC: Arcilloso. Incremento de humedad registrado tras el riego

\begin{tabular}{ccc}
\hline & Humedad del suelo $(\%)$ \\
\hline Antes del riego & Después del riego & Incremento $^{1}$ \\
\hline
\end{tabular}




\begin{tabular}{lccc}
\hline & ${ }^{* * *}$ & ${ }^{* * *}$ & \\
ARE-F & $10,13 \mathrm{~d}$ & $11,24 \mathrm{~d}$ & $1,12 \mathrm{a}$ \\
F-ARC-ARE & $13,66 \mathrm{c}$ & $14,39 \mathrm{c}$ & $0,73 \mathrm{~b}$ \\
F-ARE & $14,69 \mathrm{~b}$ & $14,98 \mathrm{~b}$ & $0,30 \mathrm{c}$ \\
F-ARC & $15,57 \mathrm{a}$ & $16,24 \mathrm{a}$ & $0,68 \mathrm{bc}$ \\
ARC & $15,52 \mathrm{a}$ & $16,43 \mathrm{a}$ & $0,91 \mathrm{ab}$ \\
\hline
\end{tabular}

ns, no significativo

${ }^{*}$ Significativo para $P<0.05$.

${ }^{* *}$ Significativo para $P<0.01 . \quad{ }^{* * *}$ Significativo para $P<0.001$.

Tabla 4 Efecto de la profundidad del suelo en el porcentaje de humedad volumétrica. Incremento de humedad registrado tras el riego

\begin{tabular}{lrcc}
\hline & \multicolumn{3}{c}{ Humedad del suelo $(\%)$} \\
\hline & Antes del riego & Después del riego & Incremento $^{1}$ \\
\hline & $\star \star \star$ & ${ }^{\star}$ & $\mathrm{ns}$ \\
$-10 \mathrm{~cm}$ & $13,52 \mathrm{c}$ & $14,43 \mathrm{~b}$ & 0,91 \\
$-20 \mathrm{~cm}$ & $13,88 \mathrm{~b}$ & $14,64 \mathrm{ab}$ & 0,77 \\
$-30 \mathrm{~cm}$ & $14,34 \mathrm{a}$ & $14,90 \mathrm{a}$ & 0,56 \\
\hline
\end{tabular}

ns, no significativo.,

${ }^{*}$ Significativo para $P<0.05$.

**Significativo para $P<0.01$., $\quad$ *** Significativo para $P<0.001$.

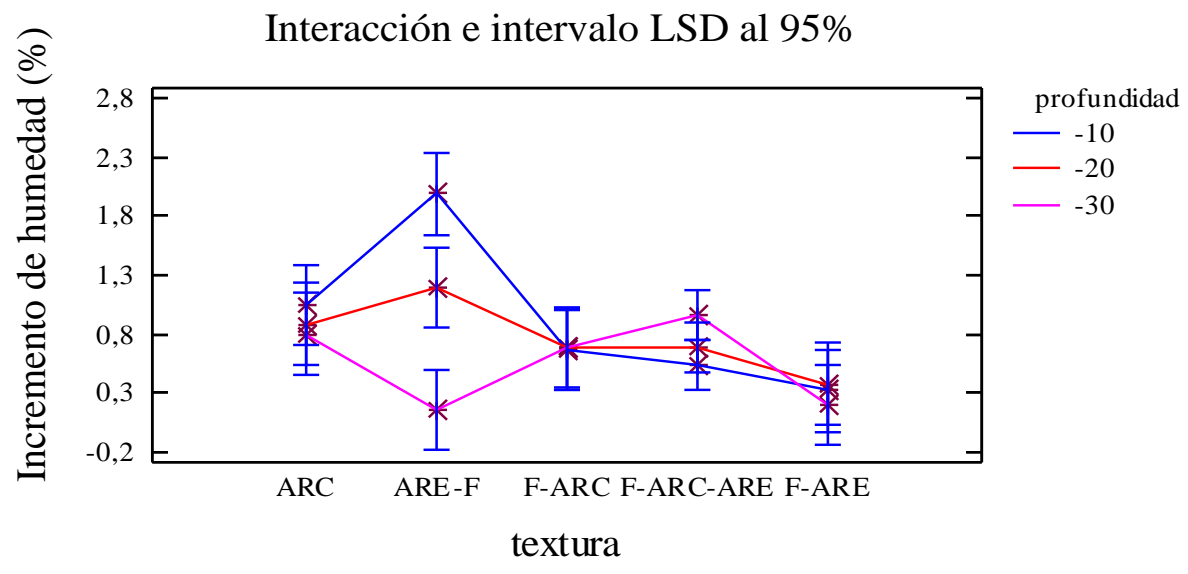

Figura 8. Interacción entre la textura y la profundidad del suelo en el incremento del porcentaje de humedad volumétrica.

En lo que respecta a la interacción existente entre la textura y la línea del muestreo (Figura 9), en suelos arcillosos se mostró un incremento del nivel de humedad mayor en la línea del gotero, con respecto a la línea intermedia. Sin embargo, en suelos francos (francoarenosos, franco-arcillo-arenosos y franco-arcillosos) no se muestran diferencias en el incremento de humedad del suelo asociadas a la línea de muestreo (Figura 9). 


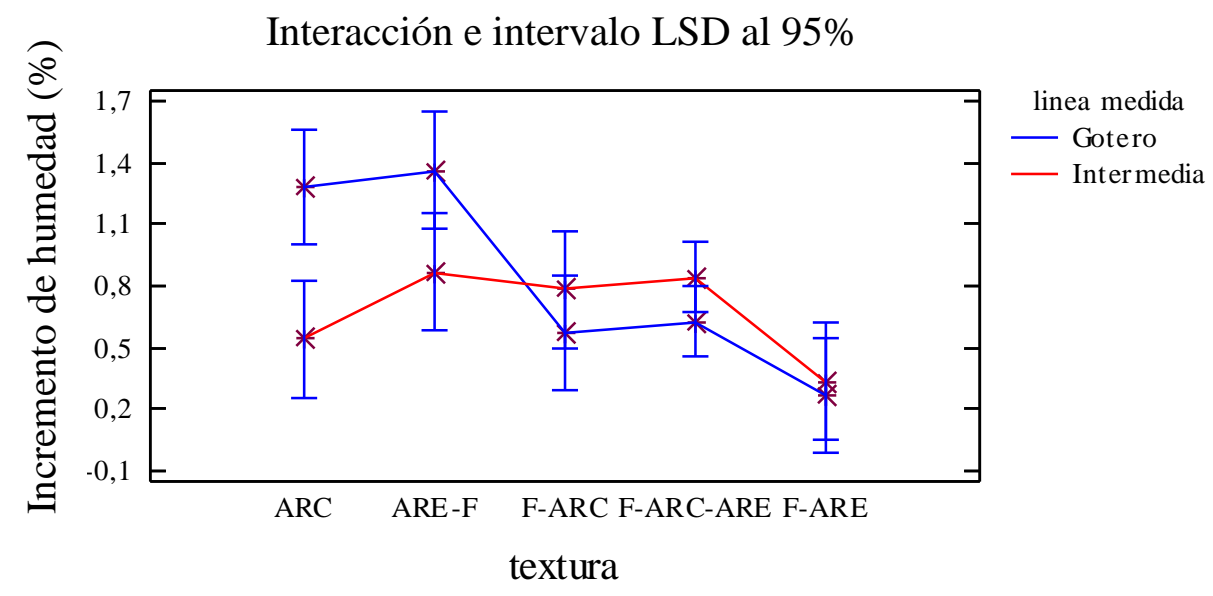

Figura 9. Interacción entre la textura y la línea de medida del suelo en el incremento del porcentaje de humedad volumétrica.

\section{Conclusiones}

A pesar de que los suelos estudiados tienen como nexo de unión el sistema enarenado, existen importantes diferencias físicas entre los mismos en aspectos como la textura, profundidad y capacidad de drenaje. exceso.

Los datos recogidos muestran que en muchas explotaciones se está regando en

Lla mayor parte de los suelos evaluados presentan los niveles de humedad más bajos en la zona más cercana al emisor, coincidente con la zona de mayor concentración radicular.

Algunos de los suelos evaluados muestran niveles de humedad más elevados en la capa inferior del suelo, lo que denota la presencia de capas inferiores impermeables, muy frecuentes en la comarca.

Los datos revelan que en la mayor parte de los casos se riega en toda la superficie del suelo. Únicamente en los suelos con porcentajes de arcilla inferiores al 15\% se aprecian zonas del terreno no humectadas.

\section{Agradecimientos}

El trabajo ha sido cofinanciado por Fondos Europeos (FSE y FEDER) y la Consejería de Agricultura y Pesca (IFAPA Junta de Andalucía) mediante el Proyecto TANSFORMACONECTA-SAR. (PP.TRA.TRA201300.10)

\section{Bibliografía y referencias}

Boswel, M.J. 1989. Manual de diseño y manejo de los sistemas de micro-irrigación, James Hardie Irrigation (Iberia), Sevilla. España.

Bresler E , 1977. Trickle-drip irrigation: principles and application to soil water management. Advances in Agronomy, 29, 343-393

Fuentes, Luis., 1998. Técnicas de Riego. $3^{\underline{a}}$ ed. Madrid, España. Ediciones Mundi-Prensa. $515 \mathrm{p}$.

Keller, J y R.D. Blisner., 2005. Sprinkle and tickle irrigation, Van Nostrand Reynhold, New York. USA. 
Lao, M.T., Jimenez, S., 2002, Los suelos enarenados en el sureste español. Vida Rural 159, $42-44$

Lubana, P.P.S., N.K. Narda, 1998, Soil water dynamics model for trickle irrigated tomatoes. Agricultural Water Management 37 145-161

Lubana, P.P.S., N.K. Narda, 2001, Modelling Soil Water Dynamics under Trickle Emitters - a Review. J. agric. Engng Res. 78 (3), 217-232 